\title{
Problems and Solutions
}

This section publishes problems and solutions believed to be new and interesting. Problems are designated by P1, P2, ..., solutions by P1S1, P1S2, .., and remarks by P1R1, P1R2, ... Correspondence regarding this section should be sent to the Problems Editor, Prof. M. A. McKiernan, Faculty of Mathematics, University of Waterloo, Ont., Canada. Authors of solutions should clearly indicate whether their paper is intended for publication in this or in the 'Research Papers' section of the journal. In case several similar solutions are received, the solutions may be edited with credits given the individual contributors.

P72S1 - J. ACZÉL

Consider the equation

and either

$$
\phi\{\phi(x, y, z), u, v\}=\phi\{x, y u, y v+z u\} \text { for all } x, y, z \in \mathbf{R},
$$

$$
\begin{aligned}
& \text { for all } y, u \in \mathbf{R} \text { or } \\
& \text { for all } y, u \in \mathbf{R} \backslash\{0\} \text {. }
\end{aligned}
$$

Results received indicate that if $\phi$ satisfies the regularity conditions

$$
\begin{gathered}
(x, z) \rightarrow \phi(x, 1, z) \text { continuous for all } x, z \in \mathbf{R}, \\
z \rightarrow \phi(x, 1, z) \text { non-constant for every } x \in \mathbf{R},
\end{gathered}
$$

then the system (1) (2) has no solution, while (1) (3) has the general solution

$$
\phi(x, y, z)=f^{-1}\{f(x)+L(y)+z / y\}
$$

for arbitrary continuous bijection $f: \mathbf{R} \rightarrow \mathbf{R}$ and arbitrary solution $L$ of

$$
L(y u)=L(y)+L(u) \text { for all } y, u \in \mathbf{R} \backslash\{0\} .
$$

Details will be published in the Research Papers section of one of the next issues of Aequationes Math.

P84 - A. R. BednareK and A. D. Wallace

Formulated in the 'Short Communication', p. 318, this issue.

Correction: Volume 4, 258 (1970). P49S1, read $r$ in place of $\pi$ 